\title{
Central Corneal Thickness Considered an Index of Corneal Hydration of the UVB Irradiated Rabbit Cornea as Influenced by UVB Absorber
}

\author{
Č. ČEJKA ${ }^{1}$, J. LUYCKX ${ }^{2}$, J. ČEJKOVÁ ${ }^{1}$ \\ ${ }^{1}$ Laboratory of Eye Histochemistry and Pharmacology, Institute of Experimental Medicine, Center \\ of Excellence, Academy of Sciences of the Czech Republic, Prague, Czech Republic, ${ }^{2}$ Laboratoires \\ Thea, Clermont-Ferrand, France
}

Received July 5, 2011

Accepted January 13, 2012

On-line April 5, 2012

\section{Summary}

UVB radiation from sunlight induces an acute corneal inflammation, photokeratitis, accompanied by changes in corneal hydration. We employed a method of ultrasonic pachymetry for daily examination of central corneal thickness as an index of corneal hydration of the rabbit cornea repeatedly irradiated by UVB radiation (312 nm, daily dose of $0.25 \mathrm{~J} / \mathrm{cm}^{2}$ during three or four days) as influenced by UVB absorber (actinoquinol combined with hyaluronic acid) dropped on the ocular surface during irradiation. One day after the third irradiation procedure the animals were sacrificed and corneas examined immunohistochemically for peroxynitrite formation, a marker of oxidative damage, the antioxidant aldehyde dehydrogenase 3A1 and endothelial nitric oxide synthase, an enzyme generated nitric oxide. Results show that UV absorber combined with hyaluronic acid protected the cornea against UVB-induced changes in corneal thickness and microscopical disturbances to the cornea (both seen after buffered saline application) until the fourth experimental day. These UVB doses are equivalent to a daily exposure of 2.5 hrs of the human cornea to solar UVB radiation for three consecutive days. It is suggested that actinoquinol/ hyaluronic acid drops might be helpful for the human eye in the defence against photooxidative and other oxidative processes.

\section{Key words}

UVB irradiation • Corneal thickness • Pachymetry

\section{Corresponding author}

J. Cejkova, Institute of Experimental Medicine, Vídeňská 1083, 14220 Praha 4, Czech Republic. Fax: +420 24106 2692. E-mail: cejkova@biomed.cas.cz

\section{Introduction}

Human exposure to UVB radiation from sunlight is increasing with ozone depletion. The ozone layer acts as a UV filter to prevent all UVC and $90 \%$ of UVB light from reaching the Earth's surface (Oliva and Taylor, 2005). The annual average ozone loss is approximately $3 \%$, primarily due to industrial pollution. It is estimated that a $1 \%$ reduction in the ozone layer leads to an increase of $0.2 \%$ to $2 \%$ in damaging UVB radiation reaching the Earth (Madronich et al. 1998). UVB radiation induces reactive oxygen species generation, which in turn causes cellular damage and a significant decrease in corneal antioxidant protective mechanisms (Rosenthal et al. 1998, Sliney 2001). Protection of the eye against the damaging effect of UVB radiation is necessary, although - fortunately - ocular exposure to UV radiation is between $2 \%$ to $30 \%$ less than surface levels (Rosental et al. 1988, Sliney 2001). This is due to the anatomic shielding of the eye (nose, cheeks, eyelids) and also personal behavior, such as wearing a brimmed hat, UV protective eyewear and other shading behaviors.

However, the cumulative daily, weekly or monthly dose of solar UV radiation reaching the human eye can vary widely as a result of various factors, such as the season, altitude, latitude, time of the year and the reflection ratio (Rosenthal et al. 1988, Sliney 1995, 1999, Sasaki et al. 2003, Sliney 2005, Sliney and Wengraitis 2006). Also, the amount of solar UV radiation reaching the human cornea varies among individual people depending on their outdoor activities as well as their personal choice of ocular protection. Zigman (1995) 
described a UVB dose of $0.105 \mathrm{~J} / \mathrm{cm}^{2}$ during a one hour exposure of the human cornea to sunlight. When we compare our daily UVB dose of $0.25 \mathrm{~J} / \mathrm{cm}^{2}$ with the dose calculated for solar UVB radiation reaching the human cornea, our dose is equivalent to 2.5 hours exposure time in sunlight. According to the later measurements of Sasaki (2009), our UVB dose of $0.25 \mathrm{~J} / \mathrm{cm}^{2}$ corresponds to 2.6 hours of solar UVB radiation reaching the human cornea.

With the aim of protecting the eye against the damaging effect of UVB radiation from sunlight during various outdoor activities, we previously examined the effect of eye drops containing an UV absorber (actinoquinol) combined with hyaluronic acid (an anionic nonsulfated mucopolysaccharide) on the rabbit cornea irradiated with $1.01 \mathrm{~J} / \mathrm{cm}^{2}$ or $0.5 \mathrm{~J} / \mathrm{cm}^{2}$ of UVB, doses equivalent to $10 \mathrm{hrs}$ and $5 \mathrm{hrs}$ (according to Zigman 1995) of solar UVB radiation reaching the human cornea, respectively (Cejka et al. 2010). We found that these drops significantly reduced the ocular disturbances evoked by the UVB doses employed. Because the protective effect of actinoquinol/hyaluronic acid eye drops was significantly increased with the decreased UVB dose (the protective effect was more pronounced after $0.5 \mathrm{~J} / \mathrm{cm}^{2}$ than after $1.01 \mathrm{~J} / \mathrm{cm}^{2}$ ), we decided to examine the efficacy of these drops on the rabbit cornea also after a UVB dose of $0.25 \mathrm{~J} / \mathrm{cm}^{2}$. In our previous study (Cejka et al. 2010) the measurement of central corneal thickness (as corneal hydration) by an ultrasonic Pachymeter was found to be a very sensitive marker of corneal damage. Therefore, after the dose of $0.25 \mathrm{~J} / \mathrm{cm}^{2}$ used in this study, the protective efficacy of actinoquinol/hyaluronic acid drops on the cornea vs. buffered saline was evaluated using this methodological approach. The measurement of central corneal thickness was completed by immunohistochemical examinations of the corneas of animals sacrificed on the fourth experimental day. Until this time point, changes of central corneal thickness were not significantly changed (compared to normal corneas) after actinoquinol/ hyaluronic acid treatment. Immunohistochemically, peroxynitrite formation, a marker of oxidative stress (Ceriello 2002, Cejkova et al. 2005, Chirino et al. 2006, Moreira et al. 2010), and the expression of aldehyde dehydrogenase 3A1 as well as endothelial nitric oxide synthase were examined in the corneas. These last two enzymes serve as sensitive markers of corneal disturbances evoked by UVB irradiation (Cejkova et al. 2005, Cejka et al. 2010).

\section{Material and Methods}

Adult New Zealand white rabbits $(2.5-3.0 \mathrm{~kg})$ were employed. The investigation was conducted according to the ARVO Statement for the Use of Animals in Ophthalmic and Vision Research. Rabbits were anesthetized by an i.m. injection of Rometar (Xylazinum hydrochloricum, Spofa, Prague, CR, $2 \%, 0.2 \mathrm{ml} / 1 \mathrm{~kg}$ body weight) and Narkamon (Ketaminum hydrochloricum, Spofa, $5 \%, 1 \mathrm{ml} / 1 \mathrm{~kg}$ body weight). The corneas of anesthetized rabbits were irradiated with a daily dose of $0.25 \mathrm{~J} / \mathrm{cm}^{2}$ for three or four days. The central corneal thickness (considered an index of hydration) was measured during the experiment using an ultrasonic Pachymeter. For immunohistochemical investigation, the corneas of animals irradiated three times and sacrificed one day later (on the fourth exprimental day) were employed.

\section{Solutions dropped on the corneal surface}

The sterile solutions, buffered solution of $0.2 \%$ actinoquinol with $0.15 \%$ hyaluronic acid, $\mathrm{pH} 7.2$ and buffered saline, $\mathrm{pH}$ 7.2, were supplied by Laboratoires Thea, Clermont-Ferrand, France.

\section{Irradiation of corneas with a UVB dose of $0.25 \mathrm{~J} / \mathrm{cm}^{2}$}

The open eyes of anesthetized rabbits were irradiated with a UVB lamp (Bioblock Scientific, Illkirch Cedex, France; $312 \mathrm{~nm}$ wavelength, $6 \mathrm{~W}$ ) at a daily dose of $0.25 \mathrm{~J} / \mathrm{cm}^{2}$ for three or four days (six rabbits in the group, the whole experiment repeated two times). During the irradiation, actinoquinol/hyaluronic acid was dropped on the right eye and buffered saline on the left eye (one drop every $30 \mathrm{~s}$, five drops in total, the first drop applied at the time 0 , one drop approximately 30 microliters). The irradiation procedure was performed with the UVB lamp positioned at a distance of $0.06 \mathrm{~m}$ for 2.5 minutes (a dose of $0.25 \mathrm{~J} / \mathrm{cm}^{2}$ ). Only the corneas were irradiated; the rest of the eye surface was protected from the UV radiation. For irradiation, a UV lamp stand - with an exactly determined distance between the lamp and the eyes - was employed. The plane of the lamp was parallel to the tangential plane of the eye (at a right angle to the optical axis of the eye). The intensity of irradiation was measured with a UVB sensor connected to a radiometer (both instruments manufactured by Cole-Parmer Inc., Vernon Hills, Illinois, USA). The total dose of irradiation was also checked using these devices. Although the source of UVB used emits only UVB rays with a peak at 
$312 \mathrm{~nm}$ (according to the irradiation spectrum given by the manufacturer), a UVC sensor (Cole-Parmer Inc.) was employed to detect any potential emission of UVC rays. After finishing the last irradiation, the animals were left without any treatment for 24 hours and sacrificed. Every day corneal hydration was measured using a Pachymeter. The corneas of normal rabbits served as controls.

The measurement of central corneal thickness (as an index of corneal hydration)

Central corneal thickness of anesthetized animals was measured using an Ultrasonic Pachymeter SP-100 (Tomey Corporation, Noritake-shinmachi, Nishi-ku, Nagoya, Japan) in the corneal center. The corneal thickness was measured every day before the irradiation procedures and on the last experimental day before sacrificing the animals. Every cornea was measured four times with the Pachymeter, and the mean value of the thickness and the standard deviation were computed.

\section{Immunohistochemical examinations}

For immunohistochemical examinations, the eyes of animals sacrificed on the fourth experimental day were employed, while the eyes of normal rabbits served as controls. The anterior eye segments were dissected out and quenched in light petroleum chilled with an acetone-dry ice mixture. Sections were cut on a cryostat and transferred to glass slides. Subsequently, the cryostat sections were fixed in acetone at $4{ }^{\circ} \mathrm{C}$ for $5 \mathrm{~min}$. For the localization of nitric oxide synthase, nitrotyrosine, and aldehyde dehydrogenase $3 \mathrm{~A} 1$, the following primary antibodies were used: monoclonal mouse anti-human endothelial nitric oxide synthase, (BD Biosciences, San Jose, CA, USA), monoclonal mouse anti-nitrotyrosine (Abcam, Cambridge, UK), mouse polyclonal anti-human aldehyde dehydrogenase (ALDH3A1). Subsequently, an anti-mouse HRP/DAB Ultravision Detection System (Lab Vision, Fremont, CA, USA) was employed as recommended by the manufacturer: hydrogen peroxide block (20 min), ultra $\mathrm{V}$ block (5 $\mathrm{min})$, primary antibody incubation $(60 \mathrm{~min})$, biotinylated goat, anti-mouse antibody incubation $(10 \mathrm{~min})$, and streptavidin peroxidase incubation (10 min). Visualization was performed using a freshly prepared DAB substrate/chromogen solution. Some sections were counterstained with Mayer's hematoxylin (Sigma). Negative controls included the omission of the primary antibody.

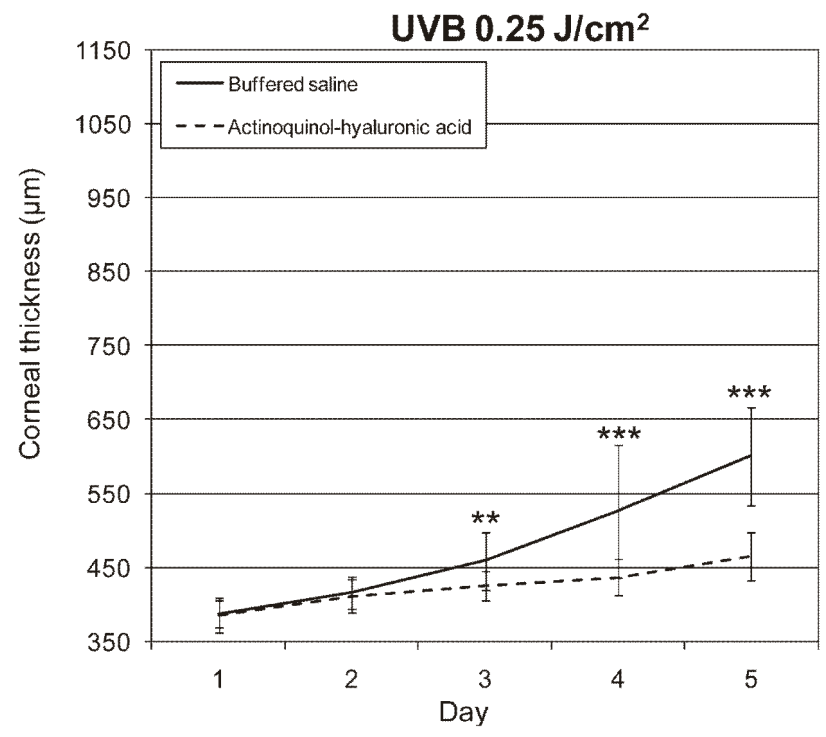

Fig. 1. Changes in corneal thickness of rabbit corneas measured by an ultrasonic Pachymeter before irradiation (day one) and at individual time points of UVB irradiation (daily dose $0.25 \mathrm{~J} / \mathrm{cm}^{2}$ ), together with the application of either actinoquinol-hyaluronic acid or buffered saline. The difference between the actinoquinol/hyaluronic acid and buffered saline-treated corneas is statistically significant on the third (** $\mathrm{p}<0.01)$, fourth $(* * * p<0.001)$ and fifth $(* * * p<0.001)$ experimental day.

\section{Image analysis and statistical evaluation}

Image analysis was carried out on stored images acquired using an Orthoplan Leitz light microscope equipped with a Leica DC 500 digital camera with Leica Image Manager (Version 4.0). The densitometric measurements of the immunohistochemical staining for enzymes (endothelial nitric oxide synthase and aldehyde dehydrogenase 3A1) were performed using KS400 (Carl Zeiss, Jena, Germany) image analysis software. For statistical analysis a one-way ANOVA test with Bonferroni's multiple comparison post-test was performed using GraphPad PRISM software (GraphPad Software, San Diego, CA, USA).

Tukey's Multiple Comparison Test was employed for evaluating the corneal thickness data using GraphPad PRISM software (GraphPad Software, San Diego, CA, USA).

\section{Results}

Central corneal thickness considered an index of corneal hydration following irradiation with a dose of $0.25 \mathrm{~J} / \mathrm{cm}^{2}$ (Fig. 1, Table 1, Table 2)

Our results show that after buffered saline treatment during the repeated irradiation of the cornea with a daily UVB dose of $0.25 \mathrm{~J} / \mathrm{cm}^{2}$, corneal thickness 
Table 1. Statistical evaluation of the gradual increase in corneal thickness (as an index of hydration) measured by an ultrasonic Pachymeter) after one, two and three repeated irradiations of the rabbit cornea with a daily UVB dose of $0.25 \mathrm{~J} / \mathrm{cm}^{2}$ and treatment with buffered saline, compared to normal corneas. Only the first irradiation of the cornea did not significantly increase the extent of corneal thickness.

\title{
Days of experiment \\ Mean thickness \pm SD \\ $P$ value (significance) normal vs. \\ irradiated
}

Tukey's Multiple Comp. Test

\author{
Day 1 (Normal) \\ Day 2 (UVB irradiated once) \\ Day 3 (UVB irradiated two times) \\ Day 4 (UVB irradiated three times) \\ Day 5 (UVB irradiated four times)
}

\author{
$386.8 \pm 17.43$ \\ $415.8 \pm 22.48$ \\ $459.0 \pm 39.05$ \\ $526.2 \pm 89.65$ \\ $600.2 \pm 66.95$
}

$\mathrm{ns}$
$* *$
$* * *$
$* * *$

Table 2. Statistical evaluation of changes in corneal thickness (measured by an ultrasonic Pachymeter) after one, two, three and four repeated irradiations of the rabbit cornea with a daily UVB dose of $0.25 \mathrm{~J} / \mathrm{cm}^{2}$ and actinoquinol/hyaluronic acid application during irradiation, compared to normal corneas. Changes in corneal thickness after irradiation and actinoquinol/hyaluronic acid treatment were not significant until the fourth experimental day (the cornea was irradiated three times).

\begin{tabular}{|ccc} 
Days of experiment & Tukey's Multiple Comp. Test \\
Mean thickness \pm SD & $\begin{array}{c}\text { P value (significance) normal vs. } \\
\text { irradiated }\end{array}$
\end{tabular}

\author{
Day 1 (Normal) \\ Day 2 (UVB irradiated once) \\ Day 3 (UVB irradiated two times) \\ Day 4 (UVB irradiated three times) \\ Day 5 (UVB irradiated four times)
}

$\begin{array}{ll}385.0 \pm 23.73 & \\ 411.2 \pm 22.21 & \mathrm{~ns} \\ 424.3 \pm 19.88 & \mathrm{~ns} \\ 436.8 \pm 23.73 & \mathrm{~ns} \\ 465.0 \pm 32.09 & * *\end{array}$

increased along with the number of repeated irradiations. Compared to buffered saline treatment, actinoquinol/ hyaluronic acid eye drops applied on the cornea during UVB irradiation suppressed the increase in corneal thickness (Fig. 1). Table 1 shows that after one irradiation (measured on day 2), the increase in corneal thickness was not significant (compared to normal corneas) following actinoquinol/hyaluronic acid as well as buffered saline application. However, after the second and third irradiation procedures (measured on days three and four), the corneal thickness was significantly increased (compared to normal corneas) after buffered saline treatment, whereas after actinoquinol/hyaluronic acid application corneal thickness was not significantly changed compared to the normal corneas (Table 2).

Differences in the corneal thickness between buffered saline application and actinoquinol/hyaluronic acid treatment were significant from the second irradiation to the end of the experiment.
Immunohistochemical examinations, image analysis and statistical evaluation (Figs 2-5)

After buffered saline treatment, an increase in the expression of endothelial nitric oxide synthase (an enzyme that generate nitric oxide) was found in the corneal epithelium (Fig. 2 b). Following actinoquinol/ hyaluronic acid application, the endothelial nitric oxide synthase (Fig. 2 c) was slightly expressed in the corneal epithelium, very similar as in the normal corneal epithelium (Fig. 2 a). In control sections in which the primary antibody was omitted from the incubation medium, no staining appeared (Fig. 2 d). Image analysis and the statistical evaluation of endothelial nitric oxide synthase expression/levels in irradiated corneas treated with actinoquinol/hyaluronic acid vs. buffered saline are shown in Fig. 3. The staining for aldehyde dehydrogenase 3A1 (an important antioxidant enzyme) after irradiation and buffered saline treatment decreased in the corneal epithelium (Fig. 4 b), whereas after actinoquinol/ hyaluronic acid application the expression 

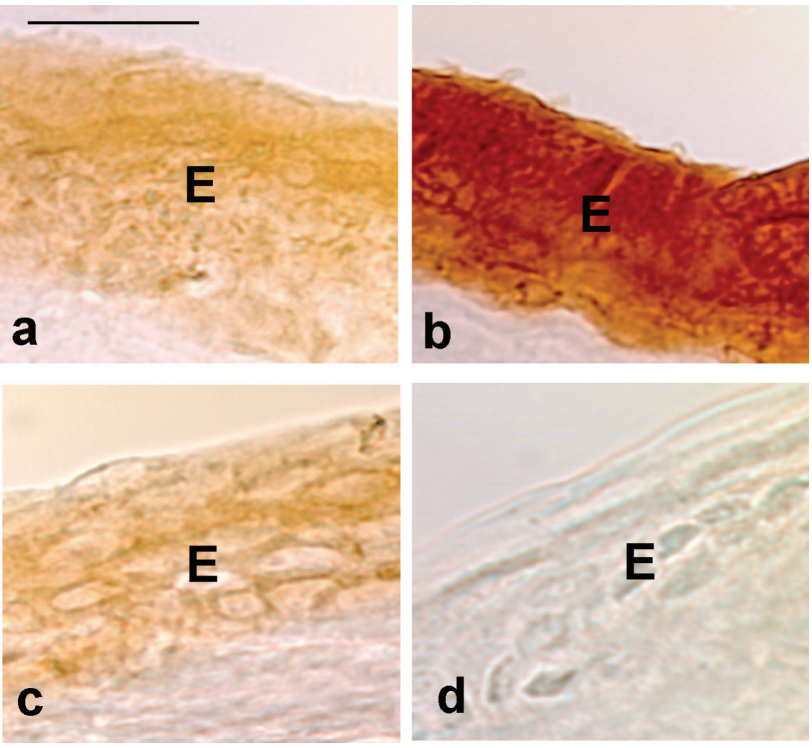

Fig. 2. The expression of endothelial nitric oxide synthase in the corneal epithelium $(E)$ of the rabbit cornea irradiated three times with a daily UVB dose of $0.25 \mathrm{~J} / \mathrm{cm}^{2}$ and treated with actinoquinol/hyaluronic acid or buffered saline. Scale bar: $10 \mu \mathrm{M}$. a - In the normal corneal epithelium endothelial nitric oxide synthase is slightly expressed. b - After buffered saline treatment the expression of endothelial nitric oxide synthase is highly pronounced in the corneal epithelium. c - Following actinoquinol/hyaluronic acid application during irradiation, endothelial nitric oxide synthase is slightly expressed in the corneal epithelium - similarly as in the normal corneal epithelium (a). d - Control section (normal corneal epithelium). The primary antibody was omitted from the incubation medium. No staining appears.

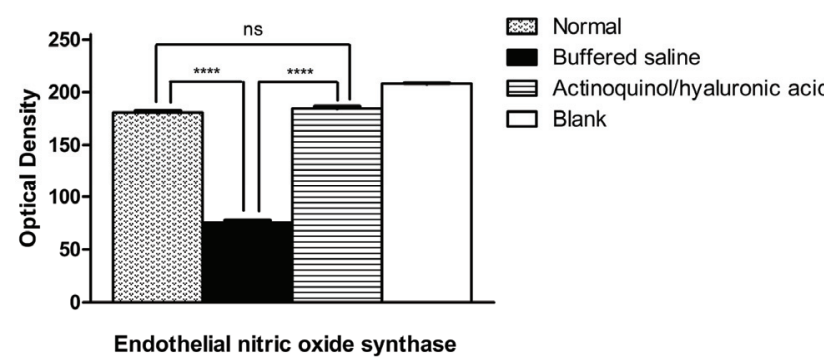

Fig. 3. Image analysis and statistical evaluation of endothelial nitric oxide synthase expression in the corneal epithelium treated with actinoquinol/hyaluronic acid or buffered saline during irradiation. Actinoquinol/hyaluronic acid treatment vs. buffered saline application: significant differences (**** $\mathrm{p}<0.0001)$. Actinoquinol/hyaluronic acid application vs. normal cornea: nonsignificant differences. Buffered saline treatment vs. normal cornea: significant differences $(* * * * p<0.0001)$.

of this enzyme was high in the corneal epithelium (Fig. 4 c) similarly as in the normal corneal epithelium (Fig. 4 a). In control stainings in which the primary antibody was omitted, no positive staining was found; only nuclei were stained by counterstaining (haematoxylin) (Fig. 4 d). Image analysis and the statistical evaluation of aldehyde dehydrogenase $3 \mathrm{~A} 1$
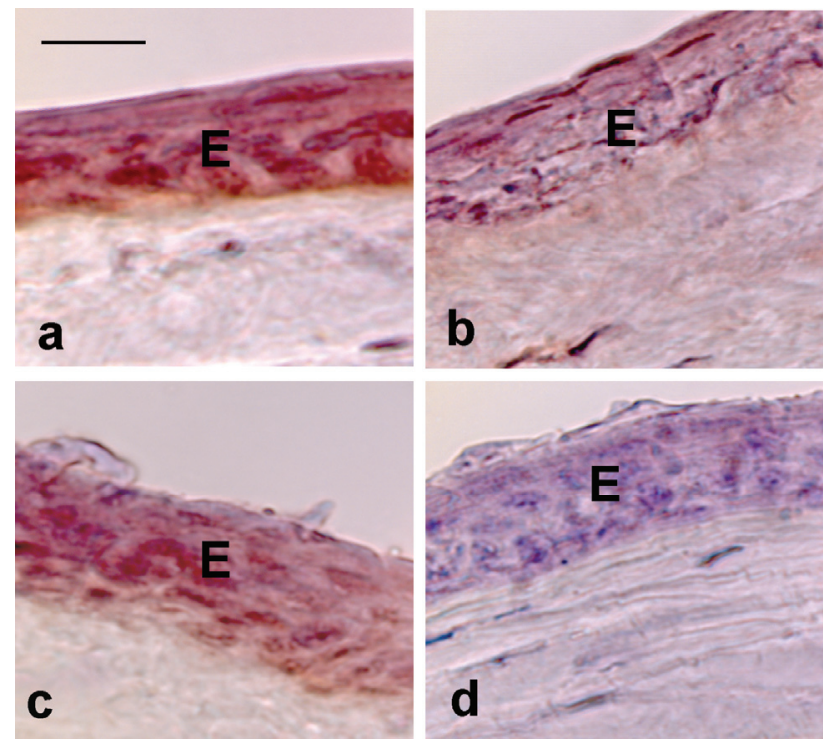

Fig. 4. The expression of aldehyde dehydrogenase $3 A 1$ in the corneal epithelium (E) of the rabbit cornea irradiated three times with a daily UVB dose of $0.25 \mathrm{~J} / \mathrm{cm}^{2}$ and treated with actinoquinol/hyaluronic acid or buffered saline. Scale bar: $10 \mu \mathrm{M}$. $\mathbf{a}$ - Aldehyde dehydrogenase 3A1 is highly expressed in the normal corneal epithelium. b - In a UVB-irradiated cornea treated with buffered saline, the enzyme expression is decreased in the corneal epithelium. c - After actinoquinol/hyaluronic acid treatment during UVB irradiation, the expression of aldehyde dehydrogenase $3 \mathrm{~A} 1$ is high in the corneal epithelium - very similar as in the normal corneal epithelium (a). d - Control staining for aldehyde dehydrogenase 3A1 (normal cornea). The primary antibody was omitted from the incubation medium. No positive staining appears. In Fig. 6 sections were counterstained with haematoxylin.

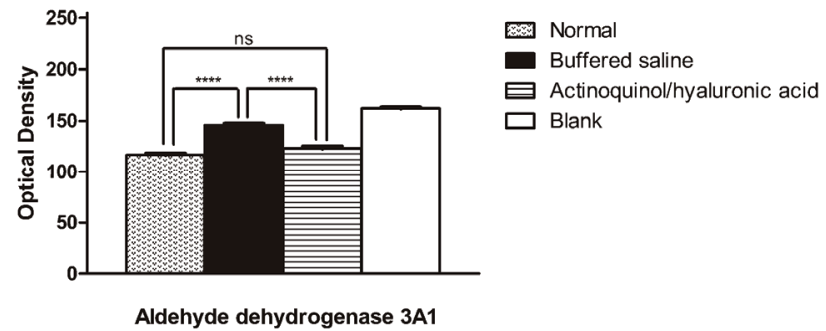

Fig. 5. Image analysis and statistical evaluation of aldehyde dehydrogenase 3A1 expression in the corneal epithelium treated with actinoquinol/hyaluronic acid or buffered saline during irradiation. Actinoquinol/hyaluronic acid treatment vs. buffered saline application: significant differences (**** $\mathrm{p}<0.0001)$. Actinoquinol/hyaluronic acid treatment vs. normal cornea: nonsignificant differences. Buffered saline vs. normal cornea: significant differences $(* * * * p<0.0001)$.

expression/levels in irradiated corneas treated with actinoquinol/hyaluronic acid vs. buffered saline are shown in Fig. 5.

Peroxynitrite formation (detected immunohistochemically by nitrotyrosine residues) is not present in the normal cornea. In irradiated corneas with low UVB dose $\left(0.25 \mathrm{~J} / \mathrm{cm}^{2}\right)$ treated with actinoquinol/ hyaluronic 


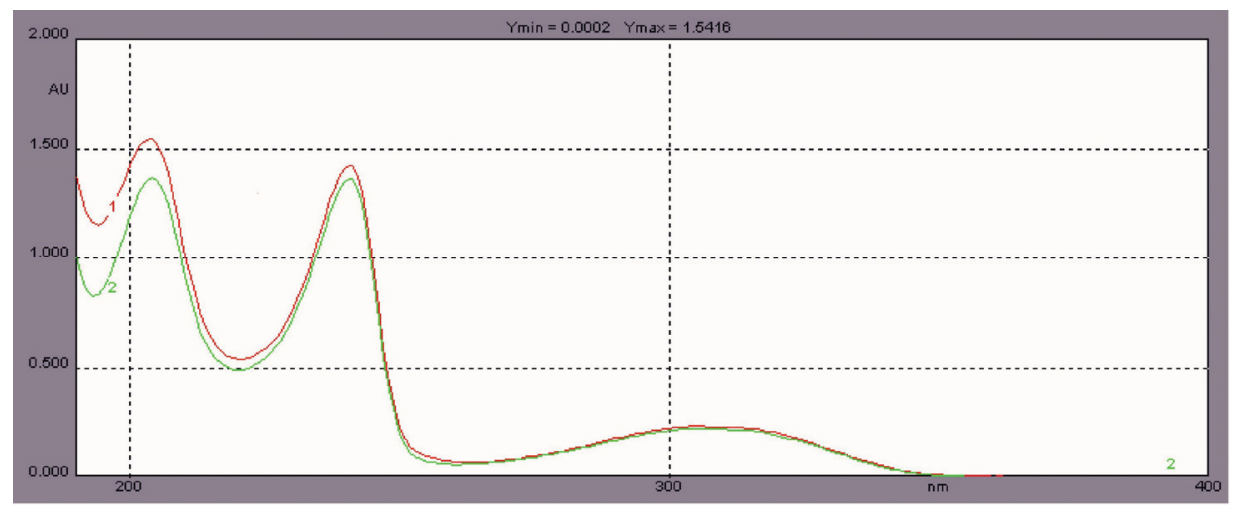

Fig. 6. In vitro UV absorbance of actinoquinol $(0.2 \%)$ in buffered saline $\mathrm{pH} 7.2$; spectrum between 190 and $400 \mathrm{~nm}$ (Curve 1). In vitro UV absorbance of actinoquinol $(0.2 \%)$ with hyaluronic acid $(0.15 \%)$ in buffered saline $\mathrm{pH} 7.2$; spectrum between 190 and 400 nm (Curve 2).

acid or buffered saline, peroxynitrite formation was also not found (data not shown).

In vitro spectrum of $U V$ absorbance of actinoquinol and actinoquinol with hyaluronic acid (Fig. 6)

Curve 1: Actinoquinol $0.2 \%$ dissolved in buffered saline $\mathrm{pH}$ 7.2, then diluted with water to obtain a legible spectrum (final actinoquinol concentration: $10 \mu \mathrm{g} / \mathrm{ml})$.

Curve 2: Actinoquinol $0.2 \%+$ sodium hyaluronate $0.15 \%$ dissolved in buffered saline $\mathrm{pH} 7.2$, then diluted with water to obtain a legible spectrum (final actinoquinol and hyaluronate concentrations: 10 and 7.5 $\mu \mathrm{g} / \mathrm{ml}$, respectively).

\section{Discussion}

Our results show that after buffered saline treatment during UVB irradiation, central corneal thickness gradually increased along with the number of repeated daily UVB doses. Actinoquinol/hyaluronic acid drops applied on the ocular surface during the irradiation significantly suppressed the gradual UVB-induced increase in corneal thickness. Comparing our present results using a daily dose of $0.25 \mathrm{~J} / \mathrm{cm}^{2}$ with those previously obtained using doses of $0.5 \mathrm{~J} / \mathrm{cm}^{2}$ and $1.01 \mathrm{~J} / \mathrm{cm}^{2}$ to investigate the efficacy of actinoquinol/ hyaluronic acid drops during repeated UVB irradiation, these drops reduced changes in corneal thickness after a dose of $0.5 \mathrm{~J} / \mathrm{cm}^{2}$ throughout the whole experiment (Cejka et al. 2010). The effect of actinoquinol/hyaluronic acid drops was small after a dose of $1.01 \mathrm{~J} / \mathrm{cm}^{2}$. However, using a dose of $0.25 \mathrm{~J} / \mathrm{cm}^{2}$, changes of corneal thickness were prevented until the fourth experimental day (Fig. 1, Table 1, Table 2).

As described previously (Cejka et al. 2010), the combination of actinoquinol (a UV absorber) with hyaluronic acid is very effective. Actinoquinol (8- ethoxyquinoline-5-sulfonic acid) a quinole, is refered to as a UV screen in the literature and is able to absorb UVB rays in vitro (Cejka et al. 2010). Hyaluronic acid is an anionic, nonsulfated glycosaminoglycan. It enhances tear film stability (Mochizuki et al. 2008) and reduces washout from the ocular surface (Nakamura et al. 2004), promotes the migration of corneal epithelial cells and accelerates the healing of corneal epithelial defects (Gomes et al. 2004). Moreover, hyaluronic acid decreases UVB-induced apoptosis and pro-inflammatory cytokine production in human corneal epithelial cell lines (Pauloin et al. 2009). According to these authors hyaluronic acid does not absorb UVB rays. This is in accordance with our results with in vitro absorbance of actinoquinol alone and actinoquinol with hyaluronic acid (Fig. 6). Therefore, the combination of hyaluronic acid with actinoquinol, absorbing UVB rays, is advantageous. In this study using a dose of $0.25 \mathrm{~J} / \mathrm{cm}^{2}$, actinoquinol/hyaluronic acid treatment during irradiation prevented changes in the expression of endothelial nitric oxide synthase (an enzyme that generate nitric oxide) as well as aldehyde dehydrogenase 3A1 (an antioxidant enzyme) in the corneal epithelium (rabbits sacrificed on the fourth experimental day) (Figs 2-5). At this time point, changes of central corneal thickness were also prevented after the application of actinoquinol/hyaluronic acid drops.

Endothelial nitric oxide synthase and aldehyde dehydrogenase 3A1 are very good markers of corneal damage (Cejkova et al. 2005, Cejka et al. 2010). The altered expression of these enzymes already points to slight corneal disturbances evoked by UVB irradiation. After a dose of $0.25 \mathrm{~J} / \mathrm{cm}^{2}$, peroxynitrite formation (detected in situ by nitrotyrosine residues) was not found in irradiated corneas treated with buffered saline or actinoquinol/hyaluronic acid drops. The elevated expression of this cytotoxic byproduct generated by the rapid reaction of nitric oxide with superoxide was found in corneas repeatedly irradiated with higher UVB doses 
(Cejkova et al. 2005).

In this study, corneal damage after UVB radiation was evaluated based on changes in central corneal thickness as an index of corneal hydration, measured using an ultrasonic Pachymeter. This method has previously been shown to be suitable for this purpose (Doughty et al. 1989, O'Donnell and Efron 2006, Cejka et al. 2007, 2010). After UVB irradiation of the rabbit cornea, the central corneal thickness increases along with an increase in corneal hydration - both tightly dependent on the daily UVB dose and the number of irradiations. While examining the effect of actinoquinol/hyaluronic acid on the rabbit cornea during irradiation with a UVB dose of $0.5 \mathrm{~J} / \mathrm{cm}^{2}$, the influence of hyaluronic acid alone (compared to actinoquinol/hyaluronic acid drops) was also investigated (Cejka et al. 2010). These authors found that the protective effect of hyaluronic acid alone on corneal thickness was negligible.

The acute corneal response to UV rays from sunlight, photokeratitis, is caused by UVB radiation (Young 2006). During this acute corneal response to UVB rays, the cornea swells. Corneal swelling appears several hours after UVB irradiation (a latent period) (Doughty and Cullen 1989) due to the damage of the corneal epithelium or endothelium, which in turns disturbs the normal corneal deturgescence mechanisms by which the cornea maintains normal (optimal) hydration and hence transparency; the corneal thickness is increased, which results in increased scatter. The cornea becomes opalescent (Cullen 2009).

According to Zigman (1995) $1.1 \mathrm{~J} / \mathrm{cm}^{2}$ of UVB radiation from sunlight reaches earth per hour at $40^{\circ} \mathrm{N}$ latitude at noon in July. This author calculated that between $0.022 \mathrm{~J} / \mathrm{cm}^{2}$ per hour ( $2 \%$ ) and $0.187 \mathrm{~J} / \mathrm{cm}^{2}$ per hour (17\%) of solar UVB radiation can impinge on the cornea. Using the mean from both extreme values, our dose of $0.25 \mathrm{~J} / \mathrm{cm}^{2}$ is equivalent to 2.5 hours exposure time in sunlight. According to recent measurements of
Sasaki (2009), our UVB dose of $0.25 \mathrm{~J} / \mathrm{cm}^{2}$ corresponds to 2.6 hours of solar UVB radiation to the human cornea (Čejka et al. 2011).

In conclusion, as evaluated by changes in corneal thickness and microscopical examinations, the UV absorber (actinoquinol) combined with hyaluronic acid protected the rabbit cornea from changes induced by UVB radiation at a dose equivalent to three repeated daily exposures of $2.6 \mathrm{hrs}$ of solar UVB radiation impinging on the human cornea. The absorption ability of actinoquinol/hyaluronic acid drops is very important not only for young people with healthy eyes performing outdoor activities in sunny weather, but also for older people who have a similar antioxidant content in their tears as do young people, but a lower tear flow rate. Therefore, older subjects have a poorer overall defense against photooxidative and other oxidative processes. This could predispose older persons to corneal stress and the development of dry eye disease (Choy et al. 2011). It is suggested that a combination of a UV absorber (actinoquinol) with hyaluronic acid (a mucopolysaccharide) in artificial tears may be helpful in humans in the protection of the eye against various harmful oxidative processes.

\section{Conflict of Interest}

PharmDr. J. Luyckx, employee of Laboratoires Thea, supplied for us the sterile solutions for dropping on the ocular surface (actinoquinol combined with hyaluronic acid, buffered saline). The authors are not aware of any conflict of interest because the preparing of sterile solutions was the only help by Laboratoires Thea.

\section{Acknowledgements}

Supported by the grant CZ: AVOZ50390512. We thank Dr. Taras Ardan for graphical and statistical help in the preparation of the manuscript.

\section{References}

ČEJKA Č, PLÁTENÍK J, GURYCA V, MICHÁLEK J, BRU゚NOVÁ B, ČEJKOVÁ J: Light absorption properties of the rabbit cornea repeatedly irradiated with UVB rays. Photochem Photobiol 83: 652-657, 2007.

ČEJKA Č, LUYCKX J, ARDAN T, ČEJKOVÁ J: The effect of actinoquinol with hyaluronic acid in eye drops on the optical properties and oxidative damage of the rabbit cornea irradiated with UVB rays. Photochem Photobiol 86: 1294-1306, 2010.

ČEJKA Č, ARDAN T, ŠIRC J, MICHÁLEK J, BENEŠ J, BRŮNOVÁ B, ROSINA J: Hydration and transparency of the rabbit cornea irradiated with UVB-doses of $0.25 \mathrm{~J} / \mathrm{cm}^{2}$ and $0.5 \mathrm{~J} / \mathrm{cm}^{2}$ compared with equivalent UVB radiation exposure reaching the human cornea from sunlight. Current Eye Res 36: 607-613, 2011. 
ČEJKOVÁ J, ARDAN T, ČEJKA Č, KOVAČEVA J, ZÍDEK Z: Irradiation of the rabbit cornea with UVB rays stimulates the expression of nitric oxide synthase-generated nitric oxide and the formation of cytotoxic nitrogen-related oxidants. Histol Histopathol 20: 467-473, 2005.

CERIELLO A: Nitrotyrosine: new findings as a marker of postprandial oxidative stress. Int J Clin Pract Suppl 129: 51$58,2002$.

CHIRINO YI, OROZCO-IBARRA M, PEDRAZA-CHAVERRI J: Role of peroxynitrite anion in different diseases. (in Spanish) Rev Invest Clin 58: 350-358, 2006.

CHOY CK, CHO P, BENZIE IF: Antioxidant content and ultraviolet absorption characteristics of human tears. Optom Vis Sci 88: 507-511, 2011.

CULLEN AP: Photobiology of the cornea. In: Photobiological Sciences Online. KC SMITH (ed), American Society for Photobiology, 2009. Available from: http://www.photobiology.info/.

DOUGHTY MJ, CULLEN AP: Long-term effects of a single dose of ultraviolet-B on albino rabbit cornea. In vivo analyses. Photochem Photobiol 49: 185-196, 1989.

GOMES JA, AMANKWAH R, POWELL-RICHARDS A, DUA HS: Sodium hyaluronate (hyaluronic acid) promotes migration of human corneal epithelial cells in vitro. Br J Ophthalmol 88: 821-825, 2004.

MADRONICH S, MCKENZIE RL, BJÖRN LO, CALDWELL MM: Changes in biologically active ultraviolet radiation reaching the Earth's surface. J Photochem Photobiol B 46: 5-19, 1998.

MOCHIZUKI H, YAMADA M, HATO S, NISHIDA T: Fluorometric measurement of the precorneal residence time of topically applied hyaluronic acid. Br J Ophthalmol 92: 108-111, 2008.

MOREIRA PL, SAYRE LM, ZHU X, NUNOMURA A, SMITH MA, PERRY G: Detection and localization of markers of oxidative stress by in situ methods: application in the study of Alzheimer disease. Methods Mol Biol 610: 419-434, 2010.

NAKAMURA S, OKADA S, UMEDA Y, SAITO F: Development of a rabbit model of tear film instability and evaluation of viscosity of artificial tear preparations. Cornea 23: 390-397, 2004.

O'DONNELL C, EFRON N: Corneal hydration control in contact lens wearers with diabetes mellitus. Optom Vis Sci 83: 22-26, 2006.

OLIVA MS, TAYLOR H: Ultraviolet radiation and the eye. Int Opthalmol Clin 45: 1-17, 2005.

PAULOIN T, DUTOT M, JOLY F, WARNET JM, RAT P: High molecular weight hyaluronan decreases UVB-induced apoptosis and inflammation in human epithelial corneal cells. Mol Vision 15: 577-583, 2009.

ROSENTHAL FS, PHOON C, BAKALIAN AE, TAYLOR HR: The ocular dose of ultraviolet radiation to outdoor workers. Invest Ophthalmol Vis Sci 29: 649-656, 1988.

SASAKI H: Sun's UV rays more dangerous in snow than on the beach. (Authorized interview given by Hiroshi Sasaki to Danielle Demetriou, Tokyo, for The Daily Telegraph), 2009. Available from: http://www.telegraph.co.uk/science/6766419/Suns-UV-rays.

SASAKI H, KAWAKAMI Y, ONO M, JONASSON F, SHUI YB, CHENG HM, ROBMAN L, MCCARTY C, CHEW SJ, SASAKI K: Localization of cortical cataract in subjects of diverse races and latitude. Invest Ophthalmol Vis Sci 44: 4210-4214, 2003.

SLINEY DH: UV radiation ocular exposure dosimetry. J Photochem Photobiol B 31: 69-77, 1995.

SLINEY DH: Geometrical assessment of ocular exposure to environmental UV radiation - implications for ophthalmic epidemiology. J Epidemiol 9: S22-S32, 1999.

SLINEY D: Photoprotection of the eye - UV radiation and sunglasses. J Photochem Photobiol B 64: 166-175, 2001.

SLINEY DH: Exposure geometry and spectral environment determine photobiological effects on the human eye. Photochem Photobiol 81: 483-489, 2005.

SLINEY DH, WENGRAITIS S: Is a differentiated advice by season and region necessary? Prog Biophys Mol Biol 92: 150-160, 2006.

YOUNG AR: Acute effects of UVR on human eyes and skin. Prog Biophys Mol Biol 92: 80-85, 2006.

ZIGMAN S: Environmental near-UV radiation and cataracts. Optom Vis Sci 72: 899-901, 1995. 